\title{
Estudio de la diversidad genética de gato doméstico (Felis catus) mediante genes asociados al color del pelaje en Lorica-Córdoba, Colombia
}

\author{
Pardo, E. ${ }^{\circledR}$; Causil, L. ' y Rodríguez, A. ${ }^{1}$ \\ 'Universidad de Córdoba. Facultad de Ciencias Básicas. Departamento de Biología. Montería. Colombia.
}

\author{
Palabras ClaVe adicionales \\ Equilibrio Hardy-Weinberg. \\ Estructura genética. \\ Flujo génico. \\ Frecuencias alélicas. \\ Heterocigosidad.
}

\section{RESUMEN}

Evaluar la diversidad y estructura genética en poblaciones de gatos domésticos (Felis catus) mediante marcadores de pelaje en Lorica, Córdoba. Se realizaron muestreos aleatorios entre los meses de agosto y octubre del año 2014, en 243 animales adultos presentes en nueve barrios de Lorica, donde se caracterizó fenotípicamente cada animal; la nomenclatura utilizada está en concordancia con el Committe Standardized Genetic Nomenclature For Cats (1968), atendiendo a los marcadores autosómicos de codificación morfológica: el locus ligado al sexo Orange (O) y los loci autosómicos Non-agouti (a), Blotched tabby (Tb), Dilution (d), Pelo largo (I) Manchado de blanco (S) y Dominante blanco (W). Los parámetros genéticos poblacionales: frecuencia alélica, diversidad genética, flujo génico, equilibrio Hardy-Weinberg y distancia genética fueron calculados a través del programa PopGene 1.31; la estructura genética se evaluó mediante el programa FSTAT v. 2.9.3.2. El marcador Manchado de blanco fue el de mayor frecuencia mientras el gen Dominante blanco presentó los valores más bajos de las frecuencias alélicas. Se registraron cifras poco significativas de variabilidad genética a nivel global y poblacional, así mismo, se obtuvo una escasa diferenciación genética entre las poblaciones, acompañado de un elevado flujo génico; se observó un exceso de heterocigotos a nivel poblacional y a nivel total, no hubo equilibrio Hardy-Weinberg en todas las poblaciones con relación a los marcadores Orange y Manchado de blanco.

\section{Study of genetic diversity of domestic cat (Felis catus) by genes associated with coat color in Lorica-Córdoba, Colombia}

SUMMARY

\section{AdDITIONAL KEYWORDS}

Hardy-Weinberg equilibrium.

Genetic structure.

Gene flow.

Allele frequencies.

Heterozygosity.

INFORMACIÓN

\section{Cronología del artículo.}

Recibido/Received: 4.4.2015

Aceptado/Accepted: 29.9.2015

On-line: 10.12 .2015

Correspondencia a los autores/Contact e-mail:

epardop@correo.unicordoba.edu.co
This study assessed the genetic diversity and population structure in populations of domestic cats (Felis catus), in Lorica (Colombia), using coat color markers. Random samples were obtained between August and October 2014, in 243 adult animals present in nine neighborhoods of Lorica. Each animal was characterized phenotypically; the nomenclature used is in accordance with Committe on Standardized Genetic Nomenclature For Cats (1968), based on markers encoding morphological variants the sex-linked orange locus Orange, autosomal loci: Non-agouti (a), Blotched tabby (Tb), Dilution (d), Long hair (I), Spotted white (S) and Dominant white (W). Population genetic parameters allele frequency, genetic diversity, gene flow, Hardy-Weinberg equilibrium and genetic distance were calculated through the program PopGene 1.31; the genetic structure was assessed using the program FSTAT v. 2.9.3.2. The Spotted white gene showed the highest frequency, while the Dominant white marker presented the lowest values of allele frequencies. Insignificant values of genetic variability and population occurred at global level, likewise, low genetic differentiation among populations, accompanied by a high gene flow was evident; an excess of heterozygotes at population and global level was observed. In no population was Hardy-Weinberg equilibrium found in relation to the Orange and Spotting white markers.

\section{INTRODUCCIÓN}

El gato doméstico (Felis catus) es un mamífero pequeño, principalmente carnívoro, pertenece a la familia de los Félidos. Es posible que haya sido resultado del cruce entre el gato montés europeo (Felis silvestris) y del gato doméstico africano (Felis lybica) en Asia y Europa. Su domesticación habría comenzado en Chipre hace unos 9500 años, donde descubren una tumba con los cadáveres de un gato y su amo. Lo cual prueba una 
Tabla I. Descripción de los 7 genes estudiados, Ruiz-García, 1994 (Description of the 7 genes studied, Ruiz-García, 1994).

\begin{tabular}{|c|c|c|}
\hline Locus & Alelos & Característica \\
\hline O (gen ligado al sexo) & $\begin{array}{l}0 \\
\mathrm{O}\end{array}$ & $\begin{array}{l}\text { Silvestre; pigmentación no naranja. } \\
\text { Mutante; toda la pigmentación es naranja; epistático para la detección del locus A. }\end{array}$ \\
\hline A (gen autosómico) & $\begin{array}{l}\text { A } \\
\text { a }\end{array}$ & $\begin{array}{l}\text { Silvestre color agouti. } \\
\text { Mutante; color no-agouti; un mismo color; color negro; epistático para la observación del locus T. }\end{array}$ \\
\hline T (gen autosómico) & $\begin{array}{l}\mathrm{t}^{+} \\
\mathrm{t}^{\mathrm{b}} \\
\mathrm{T}^{\mathrm{a}}\end{array}$ & $\begin{array}{l}\text { Silvestre; atigrado o mackerel tabby; recesivo frente a } \mathrm{T}^{\mathrm{a}} \text {, pero dominante para } \mathrm{t}^{\mathrm{b}} \text {. } \\
\text { Mutante; clásico o blotched tabby; recesivo. } \\
\text { Mutante; abisinio o abyssinian tabby; dominante; este alelo es poco frecuente. }\end{array}$ \\
\hline D (gen autosómico) & $\begin{array}{l}\text { D } \\
\text { d }\end{array}$ & $\begin{array}{l}\text { Silvestre; color denso. } \\
\text { Mutante; color diluido; recesivo. }\end{array}$ \\
\hline L (gen autosómico) & $\begin{array}{l}\mathrm{L} \\
\mathrm{I}\end{array}$ & $\begin{array}{l}\text { Silvestre; pelo corto. } \\
\text { Mutante; pelo largo; recesivo. }\end{array}$ \\
\hline S (gen autosómico) & S & $\begin{array}{l}\text { Silvestre; sin manchas blancas. } \\
\text { Mutante; manchado de blanco; dominante. }\end{array}$ \\
\hline W (gen autosómico) & $\begin{array}{l}\text { W } \\
\text { W }\end{array}$ & $\begin{array}{l}\text { Silvestre; color normal. } \\
\text { Mutante; color blanco; epistático para todos los otros colores. }\end{array}$ \\
\hline
\end{tabular}

$\mathrm{O}=$ Orange $; \mathrm{a}=$ Non-agouti $\mathrm{Tb}=$ Blotched tabby $; \mathrm{d}=$ Dilution $; \mathrm{I}=$ Pelo largo; $\mathrm{s}=$ Manchado de blanco; $\mathrm{W}=$ Dominante blanco.

relación intensa entre gatos y seres humanos; probablemente los gatos domesticados eran dedicados a actividades especiales o a personas especiales dentro de la aldea (Pennisi, 2004). El esqueleto de gato se halló sólo a 40 centímetros del ser humano. Tanto por la relativa salud del esqueleto como por el sedimento que lo rodeaba indicaría que alguien había cavado este pequeño hoyo o tumba, colocado ahí el gato y lo había cubierto rápidamente; ambos esqueletos están colocados en forma simétrica. La proximidad tan intensa de ambos en la muerte debería interpretarse como una evidencia de una fuerte relación en vida (Pennisi, 2004). Los romanos habrían introducido esta especie a Bretaña alrededor del año 300 D.C. Más tarde, serían los europeos los encargados de introducir al gato doméstico en el resto del mundo (Illanes et al., 2007; Vella et al., 2005).

La estricta relación entre las grandes movilizaciones antrópicas y la distribución actual de las poblaciones de gatos domésticos es lo que se conoce como la hipótesis de migración histórica (Ruiz-García y Álvarez, 2003). Varias poblaciones han sido analizadas desde una perspectiva de la genética de poblaciones biogeográficas en diferentes partes del mundo, utilizando las características que codifican para la longitud, distribución, coloración del pelaje y anomalías esqueléticas. La mayoría de estudios de las poblaciones de gatos se han realizado en Europa. Dado ese gran número de estudios, es sorprendente que solo algunos han incluido información sobre América Latina y específicamente de Colombia (Ruiz-García y Álvarez, 2003, 2008; RuizGarcía et al., 2005).

La variación fenotípica en la hibridación de especies o subespecies es una condición para que los ecologistas, conservacionistas y administradores de vida silvestre identifiquen poblaciones parentales y sus híbridos en el campo, por tal razón los marcadores fenotípicos, especialmente los relacionados con la coloración del pelaje, constituyen una valiosa herramienta a la hora de analizar la estructura genética de las poblaciones, debido a su gran contenido informativo, bajo costo, fácil manipulación e identificación y rápida obtención de resultados (Ruiz-García et al., 2008; Devillard et al., 2014; Adefenwa et al., 2013).

Por esta razón la presente investigación tuvo como finalidad conocer el grado de diversidad y estructura genética en poblaciones de gatos domésticos (Felis catus) mediante marcadores que codifican para el pelaje en Lorica, Córdoba, Colombia.

\section{MATERIAL Y MÉTODOS}

\section{SITIO DE ESTUDIO}

El estudio se realizó en la zona urbana de Lorica (09 $13^{\prime} 54^{\prime \prime}$ Latitud Norte y 7549'11" Longitud Oeste) y una temperatura promedio de $28^{\circ} \mathrm{C}$. Se muestrearon los barrios Nueva Colombia, San Miguel, Jesús María Lugo, La Gloria, Kennedy, 25 de Agosto, Finzenú, Los Andes y Villa Juana.

\section{OBTENCIÓN DE DATOS}

Se realizaron muestreos aleatorios entre los meses de agosto y octubre del año 2014; mediante excursiones urbanas y observación directa se realizó una clasificación fenotípica de cada uno de los individuos adultos encontrados en Lorica $(n=243)$, cada ruta se utilizó sólo una vez, a fin de evitar el remuestreo, atendiendo a la presencia o ausencia de los marcadores autosómicos: Non-agouti(a); Blotched tabby (Tb); Dilution (d), Pelo largo (1); Manchado de blanco (S) y Dominante blanco (W) y el locus ligado al sexo O (orange) (tabla I), por último se tomaron registros fotográficos de cada individuo. De igual manera, se realizó una encuesta sobre sexo, edad raza, origen y preferencias antrópicas.

\section{DISEÑO ESTADÍSTICO}

La estimación de las frecuencias alélicas de cada marcador a nivel poblacional y global, así como las medidas de diversidad genética establecidas por Nei correspondientes a la heterocigosidad esperada $(\mathrm{He})$, heterocigosidad esperada de la población total $\left(\mathrm{H}_{\mathrm{T}}\right)$, coeficiente de diferenciación genética $\left(\mathrm{G}_{\mathrm{ST}}\right)$, flujo génico $(\mathrm{Nm})$, equilibrio Hardy-Weinberg y la distancia genética entre las poblaciones, se estimaron a través del 
Tabla II. Frecuencias alélicas de cada marcador en las poblaciones estudiadas (Allele frequencies for each marker in the populations studied).

\begin{tabular}{|c|c|c|c|c|c|c|c|}
\hline \multirow[b]{2}{*}{ Poblaciones } & \multicolumn{7}{|c|}{ Locus } \\
\hline & $\mathrm{O}$ & a & $\mathrm{Tb}$ & $\mathrm{d}$ & I & $\mathrm{s}$ & W \\
\hline Nueva Colombia & 0,3939 & 0,3143 & 0,4643 & 0,4603 & 0,1224 & 0,6424 & 0,0000 \\
\hline San Miguel & 0,4118 & 0,2000 & 0,3750 & 0,5000 & 0,1538 & 0,5000 & 0,0000 \\
\hline J. Ma Lugo & 0,5000 & 0,0714 & 0,2174 & 0,7647 & 0,1250 & 0,3636 & 0,0000 \\
\hline La Gloria & 0,2353 & 0,2727 & 0,2308 & 0,3125 & 0,4000 & 0,4483 & 0,0000 \\
\hline Kennedy & 0,2473 & 01707 & 0,5333 & 0,5484 & 0,0408 & 0,6000 & 0,0000 \\
\hline 25 Agosto & 0,3333 & 0,4400 & 0,2857 & 0,4400 & 0,2000 & 0,6364 & 0,0000 \\
\hline Finzenú & 0,3208 & 0,4000 & 0,3600 & 0,5556 & 0,0556 & 0,6667 & 0,0000 \\
\hline Los Andes & 0,2727 & 0,2727 & 0,4286 & 0,3548 & 0,0404 & 0,4000 & 0,0244 \\
\hline Villa Juana & 0,4400 & 0,0909 & 0,3750 & 0,4400 & 0,1053 & 0,3846 & 0,0000 \\
\hline
\end{tabular}

$\mathrm{O}=$ Orange $; \mathrm{a}=$ Non-agouti $\mathrm{Tb}=$ Blotched tabby $; \mathrm{d}=$ Dilution $; \mathrm{I}=$ Pelo largo; $\mathrm{s}=$ Manchado de blanco; $\mathrm{W}=$ Dominante blanco.

Tabla III. Frecuencia alélica total de cada marcador fenotípico en Lorica (Total allele frequency of each phenotypic marker in Lorica).

\begin{tabular}{cccccccc}
\hline \multicolumn{1}{c}{ Locus } \\
\hline $\mathrm{O}$ & $\mathrm{a}$ & $\mathrm{Tb}$ & $\mathrm{d}$ & I & s & W \\
\hline
\end{tabular}

Total $\quad 0,3516 \quad 0,2876 \quad 0,3922 \quad 0,4949 \quad 0,1437 \quad 0,5576 \quad 0,0012$

$\mathrm{O}=$ Orange; $\mathrm{a}=$ Non-agouti; $\mathrm{Tb}=$ Blotched tabby; $\mathrm{d}=$ Dilution; $\mathrm{l}=$ Pelo largo; s= Manchado de blanco; W= Dominante blanco.

programa PopGene 1.31 (Yeh et al., 1999), la estructura genética de las poblaciones, atendiendo a los índices de fijación propuestos por Wright: $\mathrm{F}_{\mathrm{IS}}, \mathrm{F}_{\mathrm{IT}}$ y $\mathrm{F}_{\mathrm{ST}}$, se calculó mediante el programa FSTAT v. 2.9.3.2 (Goudet, 2002).

La elaboración del dendrograma que representa los valores estimados de distancia genética se realizó a través del método UPGMA (Unweighted Pair Group Methodwith Arithmetic Mean) empleando el programa MEGA 5.2 (Tamura et al., 2011).

\section{RESULTADOS}

\section{FRECUENCIAS ALÉLICAS}

Al calcular las frecuencias alélicas para cada población (tabla II) se obtuvo que el gen Manchado de blanco fue el más frecuente, especialmente en las sub- poblaciones de Finzenú ( $q=0,6667)$, Nueva Colombia $(q=0,6424)$ y 25 Agosto $(q=0,6364)$. Por su parte, el marcador Dominante blanco, responsable de la capa blanca, solo se registró en dos de las subpoblaciones: Los Andes y Villa Juana, siendo este el marcador menos frecuente en la población de Lorica. También los marcadores Non-agouti y Pelo largo mostraron bajas frecuencias, tanto a nivel subpoblacional, como a nivel global (tabla III). Cabe señalar que la frecuencia del gen Orange, fue alta $(\mathrm{p}=0,3516)$ en comparación con las reportadas en otras poblaciones por otros autores (Ruiz-García y Álvarez, 1999, 2003, 2008; Ruiz-García et al., 2005).

\section{DIVERSIDAD GENÉTICA}

En cada una de las poblaciones, el nivel medio de variabilidad genética fue alto (tabla IV) siendo la población de Nueva Colombia la de mayor heterocigosidad $(\mathrm{He}=0,3538)$, por otro lado, a nivel de marcadores, las poblaciones Jesús María Lugo, San Miguel y Villa Juana resultaron ser las de mayores índices de diversidad génica con respecto al gen Orange, sin embargo, la población 25 de Agosto, para el marcador Manchado de blanco se ubicó como la zona con el mayor grado de diversidad génica a nivel global mostrando un valor de 0,4907 , seguido por Nueva Colombia $(\mathrm{He}=0,4958)$ y Finzenú $(\mathrm{He}=0,4878)$ para el marcador antes men-

Tabla IV. Índice de diversidad genética de Nei (1973) en cada población a través de la heterocigosis esperada (He) para cada marcador (Genetic diversity index of Nei (1973) in each population through expected heterozygosity (He) for each marker).

\begin{tabular}{|c|c|c|c|c|c|c|c|c|}
\hline \multirow[b]{2}{*}{ Poblaciones } & \multicolumn{8}{|c|}{ Locus } \\
\hline & $\mathrm{O}$ & a & $\mathrm{Tb}$ & $d$ & I & $\mathrm{s}$ & W & Promedio \\
\hline Nueva Colombia & 0,4138 & 0,3735 & 0,4879 & 0,4392 & 0,2802 & 0,4824 & 0,0000 & 0,3580 \\
\hline San Miguel & 0,4430 & 0,3230 & 0,4311 & 0,4707 & 0,2800 & 0,4707 & 0,0000 & 0,3455 \\
\hline J. Ma Lugo & 0,4657 & 0,1753 & 0,3122 & 0,5080 & 0,2741 & 0,4173 & 0,0000 & 0,3053 \\
\hline La Gloria & 0,3330 & 0,3600 & 0,4560 & 0,3837 & 0,1610 & 0,4449 & 0,0000 & 0,3052 \\
\hline Kennedy & 0,3629 & 0,2619 & 0,2288 & 0,4639 & 0,2577 & 0,4748 & 0,0000 & 0,2957 \\
\hline 25 Agosto & 0,3983 & 0,4437 & 0,3712 & 0,4437 & 0,1934 & 0,4907 & 0,0000 & 0,3344 \\
\hline Finzenú & 0,3802 & 0,4190 & 0,3900 & 0,4679 & 0,2327 & 0,4878 & 0,0000 & 0,3400 \\
\hline Los Andes & 0,3594 & 0,3594 & 0,4174 & 0,4059 & 0,2220 & 0,4260 & 0,0149 & 0,3255 \\
\hline Villa Juana & 0,4437 & 0,1934 & 0,3043 & 0,4437 & 0,2176 & 0,4224 & 0,0000 & 0,2968 \\
\hline
\end{tabular}

$\mathrm{O}=$ Orange $; \mathrm{a}=$ Non-agouti $\mathrm{Tb}=$ Blotched tabby; $\mathrm{d}=$ Dilution; $\mathrm{I}=$ Pelo largo; $\mathrm{s}=$ Manchado de blanco; $\mathrm{W}=$ Dominante blanco. 
Tabla V. Heterocigosidad total para cada marcador en Lorica (Total heterozygosity for each marker in Lorica).

\begin{tabular}{lcccccccc}
\hline Locus & $\mathrm{O}$ & $\mathrm{a}$ & $\mathrm{Tb}$ & $\mathrm{d}$ & $\mathrm{I}$ & $\mathrm{s}$ & W & Promedio \\
HT & 0,4000 & 0,3232 & 0,3776 & 0,4474 & 0,2307 & 0,4573 & 0,0179 & 0,3220
\end{tabular}

$\mathrm{O}=$ Orange; $\mathrm{a}=$ Non-agouti; $\mathrm{Tb}=$ Blotched tabby; $\mathrm{d}=$ Dilution; $\mathrm{l}=$ Pelo largo; $\mathrm{s}=$ Manchado de blanco; $\mathrm{W}=$ Dominante blanco.

Tabla VI. Equilibrio de Hardy-Weinberg para los marcadores Orange (O) y Manchado de blanco (s) (Hardy-Weinberg equilibrium for Orange (O) and White spotting (s) markers).

\begin{tabular}{lcccc}
\hline Población & Locus & $\chi^{2}$ & Grados de libertad & $\mathrm{p}$ \\
\hline \multirow{2}{*}{ Lorica } & $\mathrm{O}$ & 37,8 & 1 & 0,045 \\
& $\mathrm{~s}$ & 14,4 & 1 & 0,000 \\
\hline
\end{tabular}

$\mathrm{O}=$ Orange $; \mathrm{s}=$ Manchado de blanco .

Tabla VII. Coeficiente de diferenciación genética (GST) y flujo génico (Nm) en Lorica (Coefficient of genetic differentiation (GST) and gene flow (Nm) in Lorica).

$\begin{array}{cc}\text { GST } & \mathrm{Nm} \\ 0,026 & 9,75\end{array}$

Tabla VIII. Valores de los estadísticos F para cada marcador en la población global ( $F$ statistical values for each marker in the overall population).

\begin{tabular}{lccc}
\hline Marcadores & FIT & FST & FIS \\
\hline $\mathrm{O}$ & $-0,3563$ & 0,0035 & $-0,3515$ \\
$\mathrm{a}$ & $-0,2630$ & 0,0018 & $-0,2879$ \\
$\mathrm{~Tb}$ & $-0,3444$ & 0,0013 & $-0,3924$ \\
$\mathrm{~d}$ & $-0,4936$ & 0,0008 & $-0,4978$ \\
$\mathrm{I}$ & $-0,1167$ & 0,0001 & $-0,1185$ \\
$\mathrm{~s}$ & $-0,5559$ & 0,0015 & $-0,5684$ \\
W & $-0,0050$ & 0,0088 & $-0,0140$ \\
Promedio & $-0,3049$ & 0,0025 & $-0,3186$ \\
\hline
\end{tabular}

$\mathrm{O}=$ Orange; $\mathrm{a}=$ Non-agouti; $\mathrm{Tb}=$ Blotched tabby; $\mathrm{d}=$ Dilution; $\mathrm{l}=$ Pelo largo; s= Manchado de blanco; W= Dominante blanco.

cionado, quien además mostró los niveles más altos de variabilidad genética en todas las poblaciones a excepción de Jesús María Lugo. En general, el marcador Manchado de blanco presentó el mayor valor de diversidad genética seguido de los genes Dilution y
Orange cuyo índice fue superior a los registrados por el resto de los marcadores (tabla V), en promedio el nivel de variabilidad genética en la población total fue de 0,3220 . Con relación al test de equilibrio HardyWeinberg (tabla VI), a nivel global las poblaciones reportaron ausencia de equilibrio.

\section{DiFERENCIACIÓN GENÉTICA Y FLUJO GÉNICO}

El nivel de diferenciación genética en la población de Lorica fue muy bajo (tabla VII), lo cual indica que aproximadamente el 2,6\% de la variación detectada se debe a diferencias entre las poblaciones, por lo tanto, las nueve poblaciones no fueron significativamente diferentes para los marcadores estudiados y sugiere que todas ellas se mantienen como una sola población.

En contraste, el elevado valor de flujo génico permite suponer que las poblaciones mantienen un grado considerable de intercambio genético, asumiéndose un total de nueve migrantes por generación, además la cifra obtenida resultó ser mayor que 1 y 4 , lo que indica que las poblaciones se comportan como una metapoblación.

\section{ESTRUCTURA POBLACIONAL}

Los valores negativos en cada marcador y en promedio para el estadístico $\mathrm{F}_{\text {IS }}$ (tabla VIII) evidencian un exceso de heterocigotos de los individuos con respecto a cada población y por lo tanto se asume ausencia de consanguinidad, con valores que oscilan entre $-0,0140$ para el marcador Dominante blanco y -0,5684 para el gen Manchado de blanco; con relación al estadístico $\mathrm{F}_{\mathrm{IT}}$, en promedio se obtuvo un exceso de heterocigotos de los individuos con respecto a la población total, abarcando valores de $-0,0050$ a $-0,5559$ para los marcadores Dominante blanco y Manchado de blanco respectivamente. Por otro lado el valor promedio de $\mathrm{F}_{\mathrm{ST}}$ resultó ser bajo, lo que indica la escasa diferenciación génica existente entre las poblaciones, cabe resaltar un valor similar se registró para el coeficiente $\mathrm{G}_{\mathrm{ST}}$ (tabla VII).

Tabla IX. Matriz de distancia genética (Nei, 1972) entre las poblaciones (Matrix of genetic distance (Nei, 1972) between populations).

\begin{tabular}{|c|c|c|c|c|c|c|c|c|c|}
\hline Población & 1 & 2 & 3 & 4 & 5 & 6 & 7 & 8 & 9 \\
\hline 1 & - & & & & & & & & \\
\hline 2 & 0,038 & - & & & & & & & \\
\hline 3 & 0,046 & 0,063 & - & & & & & & \\
\hline 4 & 0,041 & 0,068 & 0,094 & - & & & & & \\
\hline 5 & 0,009 & 0,040 & 0,059 & 0,063 & - & & & & \\
\hline 6 & 0,011 & 0,036 & 0,049 & 0,016 & 0,024 & - & & & \\
\hline 7 & 0,008 & 0,057 & 0,038 & 0,047 & 0,012 & 0,012 & - & & \\
\hline 8 & 0,008 & 0,040 & 0,072 & 0,039 & 0,011 & 0,013 & 0,013 & - & \\
\hline 9 & 0,006 & 0,025 & 0,051 & 0,040 & 0,019 & 0,007 & 0,014 & 0,011 & - \\
\hline
\end{tabular}

1= Nueva Colombia; 2= San-Miguel; 3= José María Lugo; 4= La-Gloria; 5= Kennedy; 6= 25-Agosto; 7 = Finzenu; 8= Los-Andes; $9=$ Villa-Juana. 


\section{DISTANCIA GENÉTICA}

La distancia genética entre las poblaciones fue baja, siendo Nueva Colombia y Villa Juana las poblaciones más cercanas (tabla IX), mientras que José María Lugo y La Gloria resultaron ser las de mayor diferencia génica, cifra poco significativa, pues no superó el $10 \%$, en este contexto, la población de José María Lugo presentó los mayores valores de distancia genética en comparación con el resto de las poblaciones.

El dendrograma (figura 1) evidencia la similitud genética entre las poblaciones de Nueva Colombia y Villa Juana a las cuales se asocia la población de 25 de agosto, luego Los Andes y Finzenú. Por otro lado, la población de José María Lugo se mantuvo alejada de las demás, pero con un valor de distancia poco significativo, que permite deducir que las poblaciones en conjunto están muy relacionadas.

A nivel de Colombia (figura 2), la población de Bogotá es la más alejada de las poblaciones estudiadas, al igual que lo es la población de Lorica en su clúster.

\section{DISCUSIÓN}

La elevada frecuencia del gen Manchado de blanco, podría estar relacionada con factores ambientales como las altas temperaturas, que posiblemente estarían favoreciendo no solo la presencia, sino el aumento de individuos portadores de dicho gen (Ruiz-Garcia et al., 2005; Shostell et al., 2005; Kaelin et al., 2012). Este hecho unido a ciertas preferencias antrópicas por cuestiones estéticas, reflejadas en la encuesta que se realizó durante el muestreo, evidencian una posible selección artificial, no solo por este marcador, sino también por el marcador Orange, de tal manera que sus frecuencias fueron elevándose progresivamente (Ruiz-García y Álvarez, 1999) al igual que la heterocigocidad esperada. Estudios han reportado que el gen Orange se ve favorecido en ambientes urbanos, cuyas densidades poblacionales son altas (Eizirik et al., 2010; Grahn et al., 2005; Rosenfeld 2010), pues tienden a sociabilizar con otros congéneres para poder co-existir y adaptarse a una mayor injerencia humana en sus vidas; lo que permite suponer que los gatos que portan este gen, están mejor adaptados a las condiciones imperantes de este sitio, que otros que no lo portan. Además, otra posible respuesta podría ser el rápido crecimiento poblacional de gatos lo cual incrementa considerablemente el flujo genético e incrementa la panmixia (Peña-Cruz et al., 2015). Cabe señalar que Ruiz-García y Álvarez (1999) proponen que la carencia del locus White puede ser utilizado como indicador de diversidad genética. En el presente estudio se obtuvo una frecuencia del alelo $\mathrm{W}$ ligeramente mayor al reportado en estudios realizados en otras poblaciones colombianas por Ruiz-García y Álvarez (1999), lo cual según ellos, puede revelar la cantidad de intervención humana en las poblaciones de gatos, lo que conduciría a mostrar en la presente investigación una injerencia mayor. Sin embargo, el hecho que la frecuencia del alelo W sea tan baja en todos los estudios, puede atribuirse a efectos pleiotrópicos sobre la audición (Geigy et al., 2007) lo cual podría causar complicaciones en los individuos, así como la muerte a una edad más temprana.

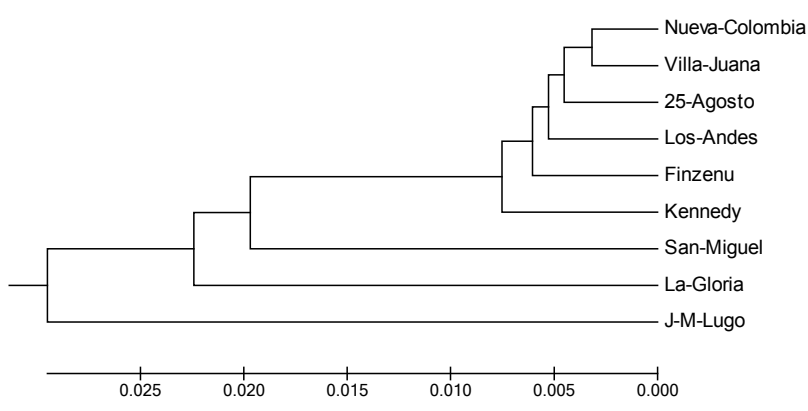

Figura 1. Dendrograma de neighbor-joining elaborados para las poblaciones de Lorica usando siete marcadores genéticos y la distancia genética (D) de Nei (1972) (Neighbor-joining dendrogram produced for populations of Lorica using seven genetic markers and genetic distance (D) of $\mathrm{Nei}$ (1972)).

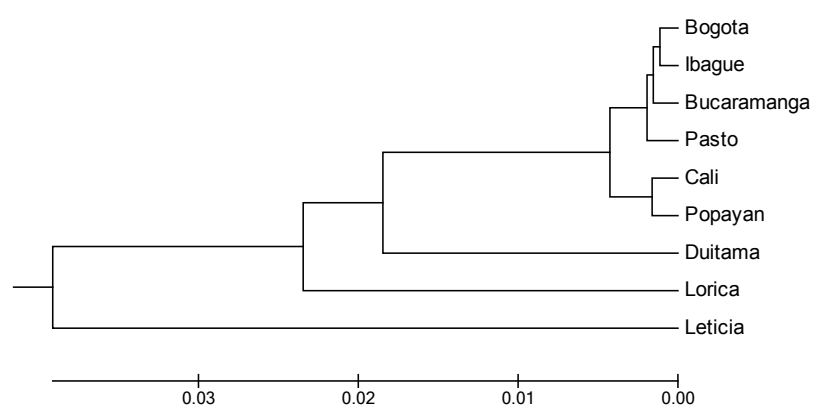

Figura 2. Dendrograma de neighbor-joining elaborados para nueve poblaciones colombianas usando siete marcadores genéticos y la distancia genética (D) de Nei (1972) (Neighbor-joining dendrogram produced for nine Colombian populations using seven genetic markers and genetic distance (D) of Nei (1972)).

La presencia de todos los marcadores estudiados en la población de gatos domésticos de Lorica, demuestra la gran variedad de genes disponibles en la zona, situación que posiblemente se puede atribuir a la ubicación geográfica estratégica de dicha población, pues desde hace mucho tiempo es paso obligado de los transeúntes que vienen desde y hacia Cartagena y poblaciones aledañas, favoreciendo el movimiento de migrantes favoreciendo un considerable flujo génico.

La desviación del equilibrio $\mathrm{H}-\mathrm{W}$ puede ser debida a diversas causas. Como los resultados arrojan un exceso de heterocigotos, podría en este caso ser atribuida a la presencia de una selección sobredominante o la ocurrencia de migraciones en las poblaciones estudiadas.

El elevado grado de flujo génico (tabla VII), permite inferir que las poblaciones se encuentran muy relacionadas genéticamente y se comportan como una metapoblación, situación a la cual se atribuye la aproximación de todas las poblaciones desde el punto de vista estructural (Ruiz-García, 1994).

El exceso de heterocigotos obtenidos a través de los distintos índices de fijación ( $\mathrm{F}_{\mathrm{IS}} \mathrm{y}_{\mathrm{IT}}$ ) en cada una de las subpoblaciones estudiadas, muestra que éstas presentan una estructura casi homogénea, hecho que sería atribuible a su cercanía geográfica, lo que ocasiona un aumento sustancial del flujo génico que hay entre las mismas, dado que la existencia de un alto intercambio 
de genes previene eventos de endogamia al interior de las poblaciones (Cortés, 2008), provocando por lo tanto un aumento de genotipos heterocigotos, en la población.

Como señala Ruiz-García y Álvarez (1999), los dueños de estas mascotas, se desplazan por toda la ciudad con éstos animales, dejando las crías o cediéndolas, así el flujo génico es alto y era de esperarse que las frecuencias alélicas de los genes evaluados no hayan diferido sustancialmente entre los muestreos realizados en Lorica, lo cual fue confirmado por el estadístico $\mathrm{F}_{\mathrm{ST}}$, ya que todas las zonas analizadas se comportan como una sola población $\left(\mathrm{F}_{\mathrm{ST}}=0,0025\right)$.

El valor de $\mathrm{F}_{\mathrm{ST}}$ junto con el de la distancia genética $\left(F_{S T}=0,0025 ; I=0,0034\right)$ obtenidas para las poblaciones estudiadas, muestran que las localidades no son subpoblaciones lo cual puede demostrarse por: el dominio de los humanos en la generación de descendientes con paternidades simultáneas, aumentando así el flujo genético dentro de las poblaciones, disminuyendo la endogamia y acrecentando la panmixia; igualmente, no existen barreras que circunscriban el flujo de los gatos, por medio de sus dueños de un lado de la ciudad al otro, es de aclarar que el valor del $\mathrm{F}_{\mathrm{ST}}$ se debe al sistema de apareamiento y no a la subdivisión poblacional. (Peña-Cruz et al., 2015).

Si se compara Lorica con otras ciudades colombianas analizadas (figura 2), se encontró que todas son semejantes genéticamente. La elevada homogeneidad genética hallada en gran parte de Colombia puede estar relacionada con el vertiginoso desplazamiento de los conquistadores españoles por el río Magdalena, los cuales establecieron muchas ciudades y villas en poco tiempo (Ruiz-García et al., 2005; Peña-Cruz et al., 2015).

La distancia de Nei (1972) mostró a Lorica comportándose genéticamente como una sola población, y al compararla con otras ciudades colombianas, se observa un gran parecido entre ellas; corroborando lo encontrado con el estadístico $\mathrm{F}_{\mathrm{ST}}$. Este gran parecido genético entre las poblaciones colombianas puede corresponder a un evento fundador común, las cuales se originaron a partir de las poblaciones españolas (Ruiz-García et al., 2005; Peña-Cruz et al., 2015), al ser una especie ausente en América hasta la inmigración de los europeos y su entrada debió estar asociada a las rutas colonizadoras de diferentes naciones europeas en el Nuevo Mundo (Ruiz-García y Álvarez, 1999).

\section{CONCLUSIONES}

El marcador con la mayor frecuencia fue Manchado de blanco, lo cual podría estar relacionado con factores como las elevadas temperaturas y las preferencias antrópicas por cuestiones estéticas, evidencian una posible selección artificial, no solo por este marcador, sino también por el marcador Orange de tal manera que sus frecuencias fueron aumentando progresivamente La presencia de todos los genes estudiados en la población de gatos de Cartagena, muestra la gran variedad de genes disponibles en la zona. La diversidad genética total encontrada en Lorica fue moderada, la mayor parte de la diversidad génica se encontró dentro de las poblaciones y poca entre las poblaciones, lo cual revela que las poblaciones locales comparten una gran proporción de la diversidad total. Se encontró además, ausencia del equilibrio de Hardy-Weinberg para los alelos O (Orange) y S (Spotting White). El dendrograma mostró a Cartagena comportándose genéticamente como una sola población, y al compararla con otras ciudades colombianas, se encontró un gran parecido entre ellas; lo cual puede corresponder a un evento fundador común al originarse estas a partir de las poblaciones españolas; además se evidenció una posible selección natural y artificial de los marcadores Orange y Manchado de blanco.

\section{AGRADECIMIENTOS}

La investigación fue financiada con recursos propios. Los autores agradecen a todas las personas en cuyas viviendas se recogieron los datos y a todos aquellos que hicieron posible la realización de este trabajo.

\section{BIBLIOGRAFÍA}

Adefenwa, M.A.; Agaviezor, B.O.; Peters, S.O.; Wheto, M.; Ekundayo, D.O.; Okpeku, M.; Oboh,B.O.; Adekoya, K.O.; Ikeobi, C.O.N.; De Donato, M.; Thomas, B.N. and Imumorin, I.G. 2013. Novel intron 2 polymorphism in the melanophilin gene is in Hardy-Weinberg equilibrium and is not associated with coat color in goats. Open $J$ Genet, 3: 195.

Committee on Standardized Genetic Nomenclature for Cats. 1968. Standardized genetic nomenclature for the domestic cat. J Hered, 59: 39-49.

Cortés, O. 2008. Análisis de la variabilidad genética en la raza bovina de Lidia utilizando información molecular. [Tesis doctoral]. Universidad Complutense de Madrid. Madrid.

Devillard, S.; Jombart, T.; Léger, F.; Pontier, D.; Say. L. and Ruette, S. 2014. How reliable are morphological and anatomical characters to distinguish European wildcats, domestic cats and their hybrids in France? J Zool Syst Evol Res, 52: 154-62.

Eizirik, E.; David, V.A.; Buckley, V.; Roolke, M.E.; Schäffer, A.A.; Hannah, S.S.; Narfström, K.; O'Brien, S.J. and Menotti-Raymond, M. 2010. Defining and mapping mammalian coat pattern genes: multiple genomic regions implicated in domestic cat stripes and spots. Genetics, 184: 267-275.

Goudet, J. 2002. FSTAT, a Program to estimate and test gene diversities and fixation indices version 2.9.3.2. http://www2.unil.ch/popgen/ softwares/fstat.htm (02/02/2015).

Grahn, R.A.; Lemesch, B.M.; Millon, L.V.; Matise, T.; Rogers, Q.R.; Morris, J.G.; Fretwell, N.; Bailey, S.J.; Batt, R.M. and Lyons, L.A. 2005. Localizing the $X$-linked orange colour phenotype using feline resource families. Anim Genet, 36: 67-70.

Geigy, C.A.; Heid, S.; Steffen, F.; Danielson, K.; Jaggy, A. and Gaillard, C. 2007. Does a pleiotropic gene explain deafness and blue irises in white cats? Vet J, 173: 548-553.

Illanes, J.; Orellana, C.; Fertilio, B.; Leyłon, V.y Venegas, F. 2007. Análisis macroscópico y microscópico del desarrollo embrionario y fetal en el gato (Felis catus), en relación con el desarrollo de la vesícula coriónica y de la placenta. Int J Morphol, 25: 467-481.

Kaelin, C.B.; Xu, X.; Hong, L.Z.; David, V.A.; McGowan, K.A.; SchmidtKüntzel, A.; Roelke, M.E.; Pino, J.; Pontius, J.; Cooper, G.M.; Manuel, H.; Swanson, W.F.; Marker, L.; Harper, C.K.; van Dyk, A.; Yue, B.; Mullikin, J.C.; Warren, W.C.; Eizirik, E.; Kos, L.; O'Brien, S.J.; Barsh, G.S. and Menotti-Raymond, M. 2012. Specifying and sustaining pigmentation patterns in domestic and wild cats. Science, 337: 1536-1541.

Nei, M. 1972. Genetic distance between populations. Amer Natur, 106: 283-292. 
Pennisi, E. 2004. Burials in Cyprus suggest cats were ancient pets. Science, 304: 189.

Peña-Cruz, A.F.; Sandoval, S.; Patiño, A.; Bedoya, M.; Rodríguez, A.; Orivela, J.; Ortega, A.; López, J.; Molina, E.; Guzmán, A.; Gil, J. and Cárdenas, H. 2015. Genetic analysis of the cat population of north and south of Cali, Colombia. Acta Biol Colomb, 20:109-116.

Rosenfeld, C.S. 2010. Animal models to study environmental epigenetics. Biol Reprod, 82 : 473-488.

Ruiz-García, M. 1994. Genetic profiles from coat genes of natural Balearic cat population: An eastern Mediterranean and North-African origin. Genet Sel Evol, 26: 39-64.

Ruiz-García, M. y Álvarez, D. 1999. Análisis filogenético de 21 poblaciones latinoamericanas de gatos mediante 10 loci morfológicos utilizando métodos de matrices de distancias genéticas y de máxima parsimonia. Bol Real Soc Esp Hist Nat, 95: 143-168.

Ruiz-García, M. y Álvarez, D. 2003. Posible origen europeo de seis poblaciones latinoamericanas de gatos y no existencia de paralelismo con el modelo colonizador británico al utilizar genes del pelaje y microsatélites. Acta Zool Mex, 89: 261-286.
Ruiz-García, M.; Álvarez, D. and Shostell, J. 2005. Population genetic analysis of cat populations from Mexico, Colombia, Bolivia, and the Dominican Republic: identification of different gene pools in Latin America. J Genet, 84: 147-171.

Ruiz-García, M. and Álvarez, D. 2008. Biogeographical population genetics perspective of the colonization of cats in Latin America and temporal genetic changes in Brazilian cat populations. Genet Mol Biol, 31: 772-782.

Shostell, J.M.; Staudinger, J. y Ruiz-García, M. 2005. Mutant alelo frecuencias en las poblaciones de gatos domésticos en Arkansas y Tennessee. J. Hered, 96: 557-565.

Tamura, K.; Peterson, D.; Peterson, N.; Stecher, G.; Nei, M. and Kumar, S. 2011. MEGA 5: Molecular evolutionary genetics analysis using maximum likelihood, evolutionary distance and maximum parsimony methods. Mol Biol Evol, 28: 2731-2739.

Vella, C.M. and Robinson, R. 2005.Robinson's genetics for cat breeders and veterinarians. 4th ed. Repr. Butterworth-Heinemann. Oxford.

Yeh, F.C.; Boyle, T.; Ye, Z. and Xiyan, J.M. 1999. POPGENE Version 1.31: Microsoft Windows-based freeware for population genetic analysis. University of Alberta and Center for International Forestry Research. 
